\title{
A BIDDING MODEL COMBINED GAME THEORY AND AI PARADIGMS
}

\author{
Tao-Sheng Wu and Ming-Teh Wang \\ Department of Civil Engineering, National Taiwan University, Taiwan, ROC \\ doxonwu@ms4.hinet.net
}

\begin{abstract}
There are many similar attributes in the behaviors of auction and construction bidding. As studied in economics, it has been widely discussed in game theory. However, game theorists may not be familiar with the subtle differences between auction and construction bidding. As an analytical framework, game theory has the merits of reducing the complication of competitive bidding, but it is still difficult to pursue answers with statistics. Since artificial neural network techniques have been frequently applied to solve ill-structured problems in many areas, they may also be applied to predict tender prices on construction bidding. To do such a prediction, this paper utilizes three methods: statistic, Neural network, and neuro-fuzzy, to compare their results. This study will address the advantages in using artificial intelligence methods.
\end{abstract}

Keywords: completive bidding model, neural network, fuzzy sets, game theory

\section{INTRODUCTION}

Many approaches have been applied to predict the bidding prices of construction projects. Competitive bidding theories have been developed for over 40 years[1][2]. Bidding prices offered by contractors and their competitors are to do with a variety of factors, which are not easily modeled and analyzed. Artificial neural network (ANN) techniques have been frequently applied to solve ill-structured problems in many areas. They can also be applied to predict the competive prices of construction bidding.

On the other hand, auction has been widely discussed in game theory[3-5]. From economists' viewpoints, their competition behaviors are very similar between the lowest competitive bidding in construction and the English first-price auction. However, when the transaction is decided, the value and quality of the commodity may not be clear to both sides in the former case, while they are clear in the later one.

Economists seldom discuss the construction bidding system directly. It was largely classified as a type of auction with multiple objectives. The highest price determines the buyer in a first-price sealed bid, whereas the lowest contractor wins the contract in construction bidding. Theoretically, these two systems are equivalent[6]. Therefore, economists tend to discuss first-price sealed bid, rather pay attention to the detail of the lowest price tendering system in construction.

In fact, the data for bidding, in many cases, is constantly changing during process. The existence of asymmetric information, in which the decision maker is unaware of his opponent's strategies, for costs and profits is apparent. How to apply data into the structure of game theory is a very difficult issue at present, although game theory approach is based on mathematics and statistical techniques. The situation of asymmetric information in construction bidding is even more difficult to handle than that in traditional auction topics.

Given that certain problems exist in applying game theory, this paper still discusses its applications to construction bidding with the following reasons:

1.The bidding game theory is suitable for modeling a strategic interdetermined issues, especially in a continuous and dynamic condition.

2.Although the approach of using game theory to model construction bidding has drawbacks, it still provides the direction for looking for optimal solutions.

3. Game theory has an extensive coverage on asymmetric information. It can be used to closely examine the information structure in construction bidding, especially the confrontation among contractors.

4. Instead of discussing economic phenomenon of the bidding behaviors, the bidding theories in construction management tend to focus on practical decisions. On the other hand, economic fields seldom discuss the bidding behaviors either. It results in lacking a proper tool for analyzing the economic conduct. To establish a comprehensive structure, the bidding game theory is still useful for the analysis of construction bidding behaviors.

However, the traditional approach, in which game theorists apply statistics for obtaining its decision parameters, needs to be modified. As proposed by 
this paper, neural network and neuro-fuzzy are good substitutes to extract information from previous cases. In parallel, this paper also uses statistics to analyze construction bidding in order to compare the effectiveness of predicting tender prices among these three approaches.

\section{BIDDING GAME}

This research utilizes the bidding game, as shown in Figure 1 by using extensive form, to describe the information structure and bidding decisions. The perforation in the figure indicates that the contractor's information at these two decision points are identical, meaning that the contractor does not know the lowest bidding price $(\mathrm{P})$ offered by his competitors and which decision point he is. Once he decides his offer of bidding price $\mathrm{B}$, the profit can be calculated accordingly.

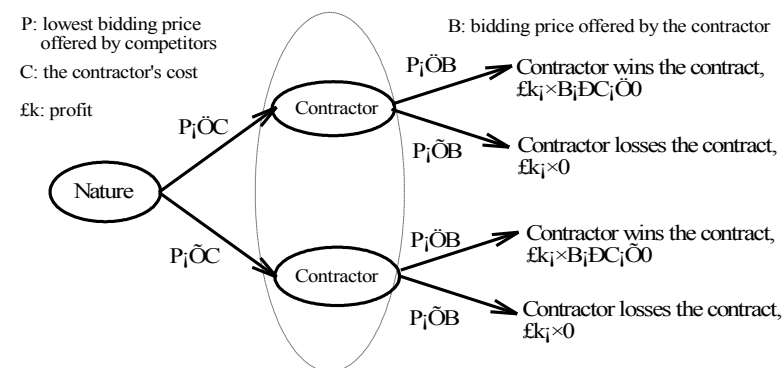

Figure 1. Bidding Game for a Contractor

The contractor needs to make a decision of his bidding price. His major considerations will be the price(or cost) possiblity density distribution. Namely, he has to consider the cost estimation distribution by each of his competitors. Therefore, when using a cost estimation generated by a fixed probability to make the decision, the more accurate the cost estimation is, the closer to the average value the cost will be.

Although the contractor does not know what the lowest bidding price $(\mathrm{P})$ his competitors can offer, he may use probability theory to define the probability for P. If the contractor's anticipation of $\mathrm{P}$ is less than his estimated cost $\mathrm{C}$, then he is facing the problem whether or not offering his bidding price less than his estimated cost.

If researchers use the game theory to examine a single contractor's bidding game, the important issues they have to consider include cost function, economic environment, owner and other contractors. Due to complex interactive relationships and possible problems of data collection, the contractor is unable to analyze these issues simply based on the individual features of his competitors. Therefore, these competitors can only be treated as a group, which is called the Nature in game theory. If a multiperson game is used as the structure for discussion, then every individual contractor's behavior must be discussed separately. However, the game will become even more complex and, therewith is more difficult to derive an answer.

Althrough there are many achievements in moden game theory, it is difficult to model the relationship between the events and their timings. If a contractor is awarded in case 1 and loses in case 2, how to model his behavior among different cases is an important issue too. If contractors will follow the awarding results to change their mutual competitive positions, this can serve as an input of bidding strategy for the next case. When a case has been awarded, it may change the market environment totally. In this case, the bidding game with theoretical analysis can be very complicated.

In terms of a bidding game with multiple contractors, and upon examining the relationship among different cases, timing factor is indicated in the horizontal axis, or in the case of a single contractor on the horizontal axis, which means the contractor's bidding memory uses time or event as the basis for refencing their orders. If bidding cases are many and frequent, the difference between time driving analysis and event driving analysis will not be serious. But if the cases are relatively few, then there will be very different.

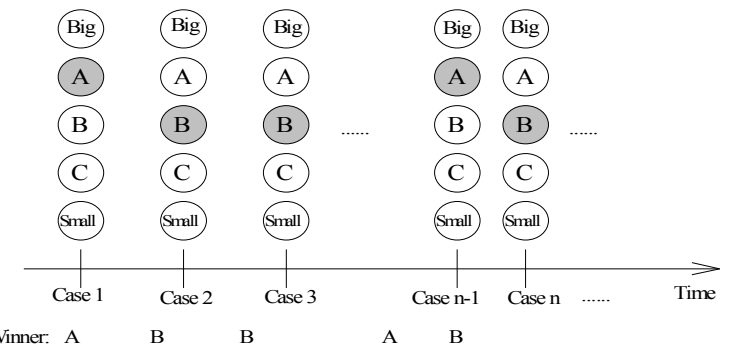

Figure 2. Relationship between Timing and Bidding Winning Cases

Figure 2 also indicates a multi-execution in a multi-contractor bidding game. The previous (or several previous times) results will have impact on the current bidding. Therefore, the information at different timings must be handled differently. As a result, a simple consideration on the opponent's probability distribution may not be suited. Moreover, assuming a fixed probability cost function is not sufficient, because the opponent may not always use the same strategy in bidding. The strategy can be changed promptly due to low winning rates recently or by any other factors. This type of asymmetric information also needs to be predicted via a proper analysis approach.

\section{PREDICTION APPROACHES}

Among the three approaches applied in this paper, the method of multiple variable statistics is most well-known to researchers. This paper does not intend to elaborate on the statistical technique. The statistical technique used here is a linear variable 
statistics analysis with SPSS ${ }^{\mathrm{TM}}$.

Neural network technique has been applied to tackle the issues on competitive bidding theory recently[7,8]. This study applies the backpropagation training method, which is widely used in neural network. The development of software for this method is quite mature. Specifically, Qnet 2.1 of Vesta Services Company is used in this study.

On the other hand, the neuro-fuzzy method has not yet been applied to the topics of the competitive bidding theory[9]. With this method, some variables that have precise values, such as price, can be numbers as accurate as down to the last single digit. In fact, the trade-off between the effectiveness and the accuracy of information in terms of strategic necessity is a topic that requires discussion and verification.

This research uses the Inform Software Company's Fuzzy Tech as the tool in generating fuzzy rules. This tool can provide basic fuzzy mathematics calculation and automatically generate the fuzzy rules needed. Moreover, the 'Neural' learning method is then utilized to determine the degree of importance on the rules. Finally, the $\alpha$-cut method can be used to simplify the rules.

The case data of the projects of Taipei City's Mass Rapid Transit System are used in this paper to verify the effect of learning and prediction of construction tender prices. In the Taipei City's MRT construction projects, the actual data items used include publicized date of tendering news, bidding date, awarded contractor, budget, total contract amount, and bidding prices by all bidders. After eliminating the cases that are under one million, the total number of the projects is 56 .

These data items include type of construction, governing agency, sponsoring agency, project name, construction site, project duration, contract budget, contract ceiling price, contracted amount, contract signing date. To examine the impact of contract tendering dates on tender prices, this study uses contract signing dates to represent the timing of this contract on the market, and to express the possible impact of time on the tender price.

\subsection{Statistics}

One other expression for measuring the prediction of tender price is to compare the difference between the actual tender price and the predicted tender price. Therefore, this paper uses tender price as a variable to conduct statistical analysis. Taking the first 49 cases for the analysis to predict the last seven cases, the analysis obtains the value of R-square (0.875), showing a weak correlation between tender prices and predicted tender prices. The average variation (average of the absolute error ratios) on the tender price prediction is $23 \%$, which indicates that the model's predictability still has room to be improved.

\subsection{Neural nets}

Utilizing neural network to implement the learning process, as this research has attempted several network structures, the effects of the prediction on tender price are good. After 100,000 times of trial runs, RMS (Root mean square) is reduced to 0.00002 .

Extracting 56 cases, this research uses the first 49 cases to implement a learning study, while the latter seven cases are used to verify prediction effect. The other group experiments are to classify these contractors into three groups: small, medium, and big. Every group's prices are treated as one; it will enhance the predictability even more.

This paper conducted the learning and prediction with the step ideal, it means we use first 49 to predict last seven, and then first 50 for last six, and so on. It simulates the behavior of a contractor who is in real decision situation.

Table 1. Results of neural network prediction for a contractor

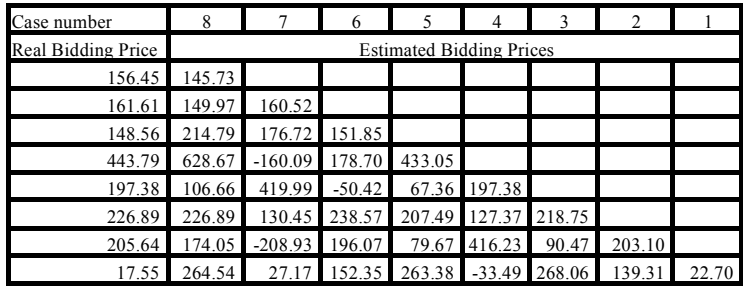

Table 2. Results of Neural network Prediction for group contractors

\begin{tabular}{|r|r|r|c|c|c|c|c|c|}
\hline Case number & \multicolumn{1}{|c|}{8} & \multicolumn{1}{|c|}{7} & 6 & 5 & 4 & 3 & 2 & 1 \\
\hline Real Bidding Price & \multicolumn{8}{|c|}{ Estimated Bidding Prices } \\
\hline-100.00 & -12.21 & & & & & & & \\
\hline-100.00 & -102.09 & 96.70 & & & & & & \\
\hline 146.50 & -63.46 & 91.94 & 138.86 & & & & & \\
\hline 413.20 & 377.41 & 420.12 & 424.00 & 438.76 & & & & \\
\hline 173.70 & 10.04 & 88.01 & 279.52 & 135.68 & 210.64 & & & \\
\hline 196.65 & -124.14 & 93.99 & -171.35 & -115.90 & -150.16 & 198.81 & & \\
\hline 175.00 & 77.34 & 354.54 & -86.73 & 6.68 & -87.48 & 273.84 & 152.80 & \\
\hline 16.80 & -179.11 & 35.05 & -231.01 & -204.52 & -229.23 & 179.85 & 345.39 & 29.88 \\
\hline
\end{tabular}

\subsection{Neuro-fuzzy}

Applying fuzzy sets to implement learning on all cases has yet provided any effective results (upon learning, the support degree of rules remains zero), since the rules acquired through the learning process are insufficient, especially the predictability on the pricing (ceiling price or tender price) is somehow low.

The reason for these problems may be due to the number of cases is too small to analyze the complex behavior. Thus, this study further groups these contractors into three and conducts another analysis. The results of the analysis are outlined in Table 3 . Among neuro-fuzzy prediction, the prediction of price/budget ratio is more accurate than that of tender price. 
Table 3. Results of Neuro-Fuzzy Prediction for group contractors

\begin{tabular}{|r|c|c|c|c|c|c|c|c|}
\hline Case number & 8 & 7 & 6 & 5 & 4 & 3 & 2 & 1 \\
\hline Price/Budget & \multicolumn{7}{|c|}{ Estimated Bidding Prices/budget } & \\
\hline 0.000 & -0.256 & & & & & & & \\
\hline 0.000 & -0.256 & 0.533 & & & & & & \\
\hline 1.085 & -0.256 & 0.533 & 0.545 & & & & & \\
\hline 0.954 & -0.256 & 0.533 & 0.545 & -0.115 & & & & \\
\hline 0.893 & -0.256 & 0.533 & 0.545 & -0.115 & 1.388 & & & \\
\hline 0.870 & -0.256 & 0.533 & 0.779 & -0.115 & 0.533 & 0.443 & & \\
\hline 0.921 & -0.256 & 0.533 & 0.545 & -0.115 & 1.388 & 0.443 & 0.448 & \\
\hline 1.772 & -0.256 & 0.533 & 0.545 & 0.560 & 1.388 & 0.229 & 0.448 & 0.699 \\
\hline
\end{tabular}

\section{INFORMATION STRUCTURE AND THE CHANGED GAME FORM}

To implement the prediction and analysis for construction tender prices, this research focuses on the data from eight contractors who have the most bidding data in attending the tendering of Taipei City's MRT construction projects.

Figure 3 shows the results of the prediction by using the above mentioned three different prediction paradigms. The horizontal axis indicates different analysis methods, while the vertical axis represents the ratio of predicted tender price to actual tender price for the last seven cases and the first few cases serve as training cases. Therefore, the three straight lines in the figure indicate for these three approaches, respectively. The length of each line in Figure 3 shows the level of closeness between the predicted value and the actual value. Neural network is found to be most accurate prediction approach.

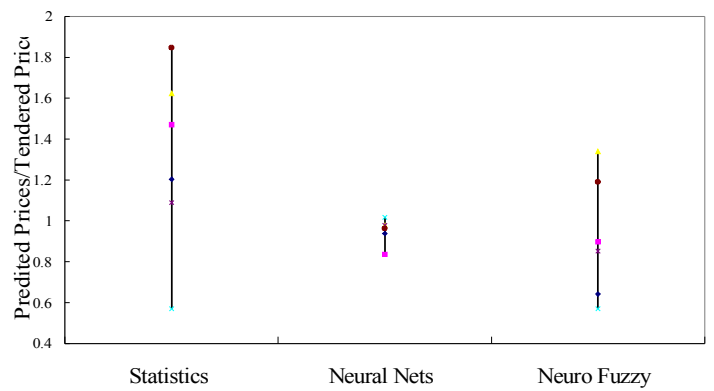

Figure 3. Comparison of Predicted Price Ratios by Using Different Methods

If the contractor's behavior on whether to bid or not can be predicted, plus that the neural network may contain certain degree of predictability on the contractor's bidding prices, then the method in applying probability to make decision may be changed. Naturally, these predictions on bidding behavior may not entirely be accurate, but it has somewhat changed the uncertainty of the opponent's decision behavior.

If the precision on this type of prediction can be accepted, then it is feasible to change the bidding game, which is once with n-person and incomplete information, into a simple bidding game with a single contractor. As shown in Figure $4, \mathrm{P}$ is the tender price, while $\mathrm{OC}$ is the contractor's opportunity cost. Before the bidding, the contractor does not know the tender price. What he knows is his own opportunity cost, yet he does not know the bidding prices and the opportunity costs of his competitors (This is a strategy with incomplete information).

$\mathrm{PP}$ is the predicted value on the lowest bidding price of the contractor, while the two crisscross straight lines in the middle represent the conditions of erroneous predictions. If the prediction with artificial intelligent approaches can reach a certain level of accuracy, then the possibility of misjudgment can be avoided. When the predicted bidding price PP is greater than his opportunity cost, the contractor may decide to proceed for winning the bidding. Otherwise, no further action will be taken for winning the bidding from this contractor. If the bidding is proceeded, then his bidding price can be derived by deducting a small number of profit allowance from the predicted lowest bidding price. The number of this small value is determined by the contractor's attitude towards risk.

On the contrary, if the analysis approach fails to provide accurate predictions, the probability of misjudgment should not be overlooked. At this time, the probabilities of all kinds of situations must be discussed, which is equal to using the probability strategy in a bidding game with multiple contractors.

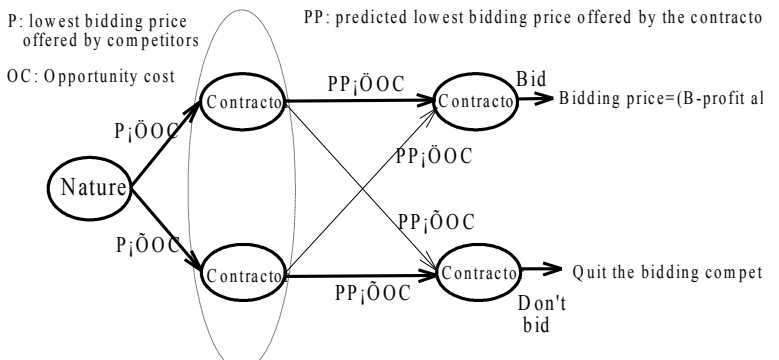

Figure 4. Contractor's Decisions of Bidding and Pricing

As a result, an accurate prediction approach not only simplifies bidding games, it but also simplifies the original decision behavior that needs to consider the probability into a simple decision issue. Yet, at this time, the possible base in determining the bidding price may stem from personal experiences, and not via a quantitative method. Therefore, if the construction management fields can precisely utilize the prediction techniques on tender prices, then the current bidding decision method led by experience can be turned to more precise decision models, which can be carefully examined.

\section{CONCLUSION}

This study utilizes the game theory's framework to analyze the strategic bidding decision of contractors. With given cases, the effectiveness of 
the predictions made by neural network and neurofuzzy is apparently better than that of statistical regression analysis. The change of information structure can result in the change of the form of the bidding game. N-person dynamic game can be solved with AI paradigms.

\section{REFERENCES}

[1]Friedman, L. (1956), A Competitive Bidding Strategy, Operations Research, Vol. 4, 104-112.

[2]Gates, M. (1967), Bidding Strategies and Probabilities, Journal of the Construction Engineering and Management, ASCE, Vol. 93, No. CO1, 75-115.

[3]Vickrey, W. (1961), Counterspeculation, Auction, Competitive Sealed Tenders, Journal of Finance, Vol. 16, No. 1, 8-37.

[4]Milgrom, P.R. \& Weber, R.J. (1982), A Theory of Auctions and Competitive Bidding, Economeirica, Vol. 50, No. 5, 1089-1122.

[5]Rasmusen, E. (1989), Game and Information - An Introduction to Game Theory, Basil Backwell Ltd., New York, 246-250.

[6]Engelbrecht-Wiggans, R. (1980), Auctions and Bidding Models: A Survey, Management Science, Vol. 26, No. 2, 119-142.

[7]Moselhi, O., Hegazy, T., and Fazio, P. (1993), DBID: Analogy-based DSS for Bidding in Construction, Journal of Construction Engineering and Management, ASCE, Vol. 119, No. 3, 464-479.

[8]Wanous, M., Boussabaine, A.H. and Lewis,J., Bid/no Bid: a parametric solution, Construction management and Economics, Vol.18, No.4, pp457-466, 2000.

[9]Li, H. and Peter E. D. Love, Combining rulebased expert systems and artificial neural networks for mark-up estimation, J. of Construction Engineering and Management, ASCE, Vol.124 (4), pp.297-304. 